\title{
Validity of Evaluation Index Utilizing Three Components of Trunk Acceleration during Walking
}

\author{
Hiroshi Osaka, RPT, MS ${ }^{1,2)}$, Koichi Shinkoda, RPT, PhD ${ }^{3)}$, Susumu Watanabe, RPT, PhD ${ }^{1)}$, \\ Daisuke Fujita, RPT, MA ${ }^{1)}$, Hiroshi Ishida, RPT, PhD ${ }^{1)}$, Kenichi KobarA, RPT, PhD ${ }^{1)}$, \\ Yosuke Yoshimura, RPT, $\mathrm{PhD}^{1)}$, Tomotaka Ito, RPT, $\mathrm{MS}^{1,2)}$ \\ 1) Department of Rehabilitation, Faculty of Health Science and Technology, Kawasaki University \\ of Medical Welfare: 288 Matsushima, Kurashiki, Okayama 701-0193, Japan. E-mail: osaka@ \\ mw.kawasaki-m.ac.jp \\ 2) Health Science Major, Division of Physical Therapy and Occupational Therapy Sciences, Hiroshima \\ University Graduate School of Health Sciences \\ 3) Biomechanics Laboratory, Applied Life Sciences, Hiroshima University Institute of Biomedical and \\ Health Sciences
}

\begin{abstract}
Purpose] The purpose of this study was to investigate the validity of an evaluation index of the vertical (VT), anteroposterior (AP), and mediolateral (ML) directions of trunk acceleration during walking. [Methods] Twenty-nine subjects volunteered for this study. Trunk acceleration and ground reaction force (GRF) during normal walking were measured. The root mean square (RMS), stride regularity (SR), and step symmetry (SS) were calculated on the basis of trunk acceleration. The displacement of COG was calculated by the double integration of GRF. The components of RMS, SS, SR, and COG were calculated in the AP, VT, and ML directions. We analyzed the correlation between RMS and COG and assessed differences among the three directions in SR and SS. [Results] We observed a significant correlation between RMS and COG in VT. SS and SR in the VT and AP directions were significantly higher than those in the ML direction. [Conclusion] These results suggest that RMS in the VT direction is useful in the gait analysis, SS and SR in the AP and VT directions were useful as indices showing the degree of deviation from normal gait.

Key words: Gait analysis, Accelerometer, Ground reaction force
\end{abstract}

(This article was submitted Aug. 21, 2012, and was accepted Sep. 30, 2012)

\section{INTRODUCTION}

The accelerometer is a readily available instrument for gait analysis. Gait abnormalities may be objectively determined by measuring movement of the periodic center of gravity (COG) with an accelerometer. This method has advantages such as simple measurement, low cost, and no restriction on location compared with gait analysis using force plates and a 3-dimensional motion analysis system. Several studies have reported the use of an accelerometer for gait analysis ${ }^{1-13)}$. In those studies, a gait index was calculated from the trunk acceleration, and gait analysis was conducted based on it.

The root mean square (RMS) is a gait index obtained from the acceleration waveform. Menz et al. ${ }^{7}$ reported increased RMS in subjects walking on irregular surfaces compared with a normal level surface. Mizuike et al. ${ }^{9)}$ reported that stroke patients' normalized RMS values were higher than those of healthy elderly adults. On account of these results, RMS values of trunk acceleration are believed to express fluctuations of the body at the walk. However, there have been very few reports on associations with COG during walking.

The auto-correlation coefficient (AC) is another index obtained from the acceleration waveform $\left.{ }^{3}, 9,11-13\right)$. This is used as an index of the degree of consistency of the trunk acceleration during walking; therefore, it reflects the regularity and symmetry of walking. AC has been reported to be useful in the evaluation of the risk of fall for elderly people, as a measure of the regularity and symmetry of gait $^{11-13)}$.

A tri-axial accelerometer is commonly used in gait analysis to measure acceleration in the vertical (VT), anteroposterior (AP) and mediolateral (ML) directions of the body ${ }^{2,4-9,11-13)}$. However, few reports that compared the characteristics of these three directions, and there are few reports on the differences of RMS and AC among the VT, $\mathrm{AP}$, and ML directions.

An evaluation index with high sensitivity to the degree of deviation from normal walking needs to be validated. This study aimed to investigate the validity of an evaluation index derived from the VT, AP and ML directions of trunk acceleration during normal walking. Moreover, the relationships of RMS and COG in the three directions were also explored. 


\section{SUBJECTS AND METHODS}

Twenty-nine young subjects volunteered for this study. All subjects were free from neurological dysfunction and musculoskeletal disorders at the time of the study. The mean \pm standard deviation age, height, and weight of the subjects was $21.4 \pm 1.7$ years, $170.2 \pm 5.3 \mathrm{~cm}$, and $64.6 \pm 7.7 \mathrm{~kg}$, respectively.

This study conformed to the Declaration of Helsinki. The protocol of this study was approved by Ethics Committee of Kawasaki University of Medical Welfare. Subjects provided their written informed consent prior to participation.

A tri-axial accelerometer (AS-TG; Kyowa Electronic Instruments Co. Ltd., Japan) was used to measure trunk acceleration along three axes during gait. The $x$-axis, $y$-axis, and the $z$-axis of the accelerometer corresponded with the ML, AP, and VT directions, respectively. The size of the accelerometer was 44 (width) $\times 44$ (length) $\times$ 20 (height) $\mathrm{mm}$. The total weight of the accelerometer was $110 \mathrm{~g}$. The acceleration signals were sampled at a rate of $100 \mathrm{~Hz}$ using an analog-to-digital converter (PCD-330B-F; Kyowa Electronic Instruments Co. Ltd., Japan) and stored in a personal computer. Acceleration signals were analyzed after processing with a $10 \mathrm{~Hz}$ low-pass filter. The accelerometer was positioned over the third lumbar vertebra $\left(\mathrm{L}_{3}\right)$, and secured to the subject using an elastic band and velcro ${ }^{2)}$

The GRF was recorded using 4 force plates (MG-1120; ANIMA Corp., Japan) located in the center of a walkway. The GRF was sampled at a rate of $100 \mathrm{~Hz}$.

The experiments were performed in a quiet room. All subjects were instructed to walk at a self-selected comfortable pace on the 10-m walkway. All of them walked barefoot. The accelerometer and force platform were synchronized, and the trunk acceleration and GRF during normal gait were measured.

The acceleration signals were transformed to give a mean of zero; thus, RMS was synonymous with the standard deviation. RMS was used to provide an indication of the average magnitude of acceleration in each direction during walking ${ }^{7)}$, and reflected the fluctuation of in gait performance. The RMS is time-series data of the acceleration. For the RMS calculation of three measurements we extracted at random 256 points in the measurements synchronized with the GRF signals. The average value was adopted as a representative value. RMS in the VT, AP and ML directions is presented as VT-RMS, AP-RMS and ML-RMS, respectively.

The stride regularity (SR) is a time-series characteristic of the acceleration. SR is indicated by $\mathrm{AC}$ in one gait cycle of trunk acceleration during walking ${ }^{3,11-13)}$. The AC value is a measure of the repeatability of a signal shape or waveform. AC normalizes the correlation to a dimensionless value ranging from -1 to +1 . The value nearer to 1 for two waveforms indicates a higher degree of coincidence ${ }^{14}$ ). SR was used to provide an indication of the regularity of the walking. The duration of a stride is the interval between 2 sequential initial floor contacts by the same limb (i.e., right-heel contact and the next right-heel contact) ${ }^{17)}$. From the trunk acceleration measured during walking in a steady state, SR was calculated at random for 2 consecutive strides.
The average value of three measurements was adopted as the representative value. SR in the VT, AP and ML directions is presented as VT-SR, AP-SR and ML-SR, respectively.

The step symmetry (SS) is a time-series characteristic of the acceleration. SS is represented by AC in one step of trunk acceleration during the walking ${ }^{3,11-13)}$. SS was used to provide an indication of the symmetry of alking. The interval between heel contact by each foot is a step (i.e., right and then left); there are 2 steps in each stride ${ }^{17}$. From the trunk acceleration measured during walking in a steady state, SS was calculated at random for 2 consecutive steps. The average value of three measurements was adopted as the representative value. SS in the VT, AP and ML directions is presented as VT-SS, AP-SS and ML-SS, respectively.

The displacement of COG is spatiotemporal data derived from GRF. The displacement of COG was computed by double integration of the acceleration calculated from the GRF data of 1 stride $^{15,16)}$. The acceleration in the ML and AP directions $\left(\alpha_{x}\right.$ and $\left.\alpha_{y}\right)$ were calculated as

$$
\alpha_{x, y}=F_{x, y} / m
$$

where $F_{x}$ and $F_{y}$ are GRF in the ML and AP directions, respectively, and $m$ is the body mass of the subject. The acceleration in the VT direction $\left(\alpha_{z}\right)$ was calculated as

$$
\alpha_{z}=F_{z} / m-g
$$

where $F_{z}$ is GRF in the VT direction, and $g$ is the acceleration due to gravity. The COG displacement range was determined as the difference between the maximum and minimum displacements of COG. The average value of three measurements was adopted as the representative value. COG in the VT, AP and ML directions is presented as VT-COG, AP-COG and ML-COG, respectively.

The correlation of RMS and COG in the VT, AP, and ML directions was determined by the Pearson correlation coefficient. One-way repeated-measures analysis of variance (ANOVA) was utilized to assess differences among the VT, $\mathrm{AP}$, and ML directions in SR and SS. Post hoc analysis was performed with Tukey's test. A p-value of $<0.05$ was considered statistically significant. All statistical analyses were performed using SPSS 17.0 J for Windows (SPSS Japan Inc., Japan).

\section{RESULTS}

The mean \pm standard deviation values of VT-RMS, AP-RMS, and ML-RMS were $31.8 \pm 6.3,25.9 \pm 3.9$, and $20.1 \pm 4.8$, respectively; those of VT-COG, AP-COG, and ML-COG were $3.3 \pm 0.6 \mathrm{~cm}, 2.3 \pm 0.4 \mathrm{~cm}$, and $3.9 \pm 0.7 \mathrm{~cm}$, respectively (Table 1 ). We found a significant positive correlation between RMS and COG in the VT direction ( $r=$ $0.739, \mathrm{p}<0.01)$. In the AP and ML directions, there were no significant correlations (AP: $r=0.124, \mathrm{p}=0.522$; ML: $r=$ $-0.239, \mathrm{p}=0.221)$.

The mean \pm standard deviation values of VT-SR, AP-SR, and ML-SR were $0.82 \pm 0.09,0.79 \pm 0.12$, and $0.61 \pm 0.17$, respectively (Table 2); those of VT-SS, AP-SS, and ML-SS 
Table 1. RMS and COG

\begin{tabular}{cccc}
\hline & RMS & COG $(\mathrm{cm})$ & $\mathrm{r}$ \\
\hline VT & $31.8 \pm 6.3$ & $3.3 \pm 0.6$ & $0.74^{*}$ \\
AP & $25.9 \pm 3.9$ & $2.3 \pm 0.4$ & 0.12 \\
ML & $20.1 \pm 4.8$ & $3.9 \pm 0.7$ & -0.24 \\
\hline
\end{tabular}

Mean \pm SD. VT: vertical direction. AP: anteroposterior direction. ML: mediolateral direction. RMS: root mean square. COG: displacement of center of gravity. $r$ : Pearson correlation coefficient. *: $\mathrm{p}<0.05$.

were $0.84 \pm 0.07,0.81 \pm 0.07$, and $0.63 \pm 0.12$, respectively. SR and SS in the VT and AP directions were significantly higher than that in the ML direction $(\mathrm{p}<0.05)$.

\section{DISCUSSION}

This study was designed to acquire fundamental knowledge for gait analysis, and to compare gait indices in the VT, AP, and ML directions, using tri-axial accelerometer data.

RMS indicates the fluctuation in gait performance measured by an accelerometer. It has been reported that RMS of walking on irregular surfaces is greater than that on a normal level surface ${ }^{7}$, and that stroke patients' normalized RMS values are higher than those of healthy elderly adults ${ }^{9}$. In this study, to compare RMS in the VT, AP, and ML directions, we evaluated the correlation between the displacement of COG and RMS, and it showed a significant positive correlation only in the VT direction. The mean displacement of COG in the ML and VT directions has been reported as $3.5 \pm$ $0.9 \mathrm{~cm}$ and $3.2 \pm 0.8 \mathrm{~cm}$, respectively ${ }^{17}$, which are consistent with the respective values computed from GRF in the present study. Minimizing COG displacement is a strategy for decreasing muscle activity during walking by humans, and leads to reduction of energy consumption. VT-RMS is believed to reflect the extent of COG displacement while showing the fluctuation in gait performance and can, therefore, be considered an energy-consumption index.

In this study, we also investigated SR and SS as indices of the regularity and symmetry of walking, as reported by Auvinet et al. ${ }^{3)}$ and Moe-Nilssen et al. ${ }^{11,12)}$. SR and SS are the ACs showing the degree of consistency of the acceleration across every gait cycle and every step, respectively; regularity and symmetry are greatest at values closest to 1 . The gait of humans in a steady state shows regular rhythmic leg movement ${ }^{18)}$. Dynamic electromyography data, joint moment and GRF measured by a 3-dimensional motion analysis system, during walking are cyclic with minimal change ${ }^{19,20)}$. Accordingly, normal gait in the steady state is considered as having a high regularity and symmetry. In the present study, we assessed differences in SR and SS among the VT, AP, and ML directions. SR and SS in the VT and AP directions were significantly higher than in the ML direction. Therefore, we consider SR and SS of VT and AP reflect the regularity and symmetry of normal gait, respectively.

Kabada et al. ${ }^{20)}$ found that GRF in the VT and AP directions was more repeatable than that in the ML direction, and the joint moment in the sagittal plane had greater reproduc-
Table 2. SR and SS

\begin{tabular}{cccc}
\hline & VT & AP & ML \\
\hline SR & $0.82 \pm 0.09$ & $0.79 \pm 0.12$ & $0.61 \pm 0.17^{\mathrm{ab}}$ \\
SS & $0.84 \pm 0.07$ & $0.81 \pm 0.07$ & $0.63 \pm 0.12^{\mathrm{ab}}$ \\
\hline
\end{tabular}

Mean \pm SD. VT: vertical direction. AP: anteroposterior direction. ML: mediolateral direction. SR: Stride Regularity. SS: Step Symmetry. a: Significantly different from VT by Tukey's test $(p<0.05)$. b: Significantly different from AP by Tukey's test $(\mathrm{p}<0.05)$.

ibility in the gait cycle than those of the frontal and transverse planes. Moreover, Winter ${ }^{19)}$ reported that the GRF in the VT direction had lower variability of measurement than GRF in the ML direction. The accuracy of measurement in the VT direction may be high compared with that in the AP and ML directions in kinematic analysis of the body during walking. In this study, we found significant correlation of RMS with displacement of COG, only in the VT direction. SS and SR in the VT and AP directions were significantly higher than in the ML direction. Although the results were not significant, SR and SS had the highest values in the VT direction. Moe-Nilssen et al. ${ }^{11)}$ compared trunk acceleration variability assessed by an AC computation, between fit elderly and frail elderly subjects. They reported that SR of the VT and AP directions were high in the fit group, and those in the frail group lower values, suggesting that these indices express the feature of a frail group. However, the trunk acceleration in the ML direction shows the reverse result, the walk index of trunk acceleration in the ML direction may not reflect the regularity of gait. The degree of consistency of acceleration in the ML direction with GRF was markedly low, consistent with our previous study ${ }^{21)}$. Yack et al. ${ }^{6}$ reported the inaccuracy of trunk-acceleration measurement in the ML direction during the gait. For the trunk acceleration measurement during walking, Kavanagh et al. ${ }^{1)}$ reported that rotation causes an error of measurement. An accelerometer can detect the complex motion of rotation, not only in the ML direction, but also the vertebral column. Considering previous results and those of the present study, we think that the trunk acceleration index of the ML direction is lacking in reliability as an index of the regularity and symmetry of walking, and trunk acceleration in the VT direction reflect the action of the body most accurately.

In conclusion, RMS calculated from the acceleration in the VT direction reflects the magnitude of COG displacement during walking. Moreover, SR and SS of trunk acceleration in the VT and AP directions are appropriate as indices of the regularity and symmetry of gait in a steady state. Therefore, in gait analysis with an accelerometer, it is useful to examine the characteristics of the walk indices obtained from trunk acceleration in all three directions.

There were several limitations of this study. Only healthy subjects were evaluated in this research and different results may be expected for abnormal gait or unusual postures due to illness. This study showed that body acceleration in the ML direction is less reliable than that in the VT and AP directions as an indicator of a normal walking. However, 
Moe-Nilssen et al. ${ }^{11)}$ suggested that ML direction trunk variability represents a different aspect of motor control. Further investigation is necessary to clarify the characteristics of trunk acceleration in the ML direction.

\section{REFERENCES}

1) Kavanagh JJ, Menz HB: Accelerometry: a technique for quantifying movement patterns during walking. Gait Posture, 2008, 28: 1-15. [Medline] [CrossRef]

2) Moe-Nilssen R: Test-retest reliability of trunk accelerometry during standing and walking. Arch Phys Med Rehabil, 1998, 79: 1377-1385. [Medline] [CrossRef]

3) Auvinet B, Berrut G, Touzard C, et al.: Reference data for normal subject obtained with an accelerometric device. Gait Posture, 2002, 16: 124-134 [Medline] [CrossRef]

4) Kavanagh JJ, Barrett RS, Morrison S: Upper body accelerations during walking in healthy young and elderly men. Gait Posture, 2004, 20 291-298. [Medline] [CrossRef]

5) Latt MD, Menz HB, Lord SR: Walking speed, cadence and step length are selected to optimize the stability of head and pelvis accelerations. Exp Brain Res, 2008, 184: 201-209. [Medline] [CrossRef]

6) Yack HJ, Berger RC: Dynamic stability in the elderly: identifying a possible measure. J Gerontol, 1993, 48: M225-230. [Medline]

7) Menz HB, Lord SR, Fitzpatrick RC: Acceleration patterns of the head and pelvis when walking on level and irregular surfaces. Gait Posture, 2003, 18: 35-46. [Medline] [CrossRef]

8) Zijlstra W, Hof AL: Assessment of spatio-temporal gait parameters from trunk accelerations during human walking. Gait Posture, 2003, 18: 1-10. [Medline] [CrossRef]

9) Mizuike C, Ohgi S, Morita S: Analysis of stroke patient walking dynamic using a tri-axial accelerometer. Gait Posture, 2009, 30: 60-64. [Medline] [CrossRef]

10) Itoh $\mathrm{Y}$, Miyamoto $\mathrm{M}$, Hojo $\mathrm{T}$, et al : Convenient use of accelerometer for gait analysis. Bull Osaka Med Coll, 2008, 54: 21-31.

11) Moe-Nilssen R, Helbostad JL: Interstride trunk acceleration variability but not step width variability can differentiate between fit and frail older adults. Gait Posture, 2005, 21: 164-170. [Medline] [CrossRef]

12) Moe-Nilssen R, Helbostad JL: Estimation of gait cycle characteristics by trunk accelerometry. J Biomech, 2004, 37: 121-126. [Medline] [CrossRef]

13) Bautmans I, Jansen B, Keymolen BV, et al.: Reliability and clinical correlates of 3D-accelerometry based gait analysis outcomes according to age and fall-risk. Gait Posture, 2011, 33: 366-372. [Medline] [CrossRef]

14) Winter DA: Biomechanics and motor control of human movement (4th ed). Hoboken: John Wiley \& Sons, 2009, pp 14-44.

15) Iida H, Yamamuro T: Kinetic analysis of the center of gravity of the human body in normal and pathological gaits. J Biomech, 1987, 20: 987-995. [Medline] [CrossRef]

16) Lee CR, Farley CT: Determinants of the center of mass trajectory in human walking and running. J Exp Biol, 1998, 201: 2935-2944. [Medline]

17) Perry J, Burnfield JM: Gait analysis, normal and pathological function (2nd ed). Thorofare: SLACK, 2010, pp 1-47.

18) Pailhous J, Bonnard M: Steady-state fluctuations of human walking. Behav Brain Res, 1992, 47: 181-189. [Medline] [CrossRef]

19) Winter DA: Kinematic and kinetic patterns in human gait: variability and compensating effects. Hum Mov, 1984, 3: 51-76. [CrossRef]

20) Kadaba MP, Ramakrishnan HK, Wootten ME, et al.: Repeatability of kinematic, kinetic, and electromyographic data in normal adult gait. J Orthop Res, 1989, 7: 849-860. [Medline] [CrossRef]

21) Osaka H, Watanabe S, Fujita D, et al.: Appropriate location of an accelerometer for gait analysis: A comparative study based on cross-correlation coefficient. Rigakuryoho Kagaku, 2011, 26: 785-789 (in Japanese). [CrossRef] 